\title{
Modelo de elementos finitos del hombro: comparación de los esfuerzos mecánicos de un hombro sano y un hombro con síndrome del manguito rotador
}

\author{
pags $42-50$ \\ Grupo de Investigación: Diseño Avanzado \\ Línea de investigación: Modelado computacional \\ Hector Alfonso Castro Abrile, Ana Ramírez"• \& Luis Silva»o•
}

Recibido: 5 de junio de 2015

\section{RESUMEN}

El síndrome del manguito rotador es la patología de hombro de mayor incidencia en Colombia, siendo el tendón del supraespinoso el más afectado. El objetivo de este estudio es desarrollar un modelo numérico del hombro, para determinar la variación los esfuerzos mecánicos en un hombro sano, y en otro con el síndrome del manguito rotador, debido a que en Colombia no hay estudios que permitan determinar dichos esfuerzos. Se desarrolló un modelo en tres dimensiones del hombro, a partir de un conjunto de imágenes por tomografía axial computarizada. Este modelo incluye el húmero, la escápula, el ligamento glenohumeral, los tendones del manguito rotador y el cartílago articular. Se analizaron los modelos mediante el método de elementos finitos, y se obtuvieron los esfuerzos de Von Mises. Posteriormente, se compararon los resultados obtenidos de un hombro sano, y de un hombro con síndrome del manguito rotador, para saber cómo varía la distribución de los esfuerzos mecánicos. Luego se compararon los resultados obtenidos en este estudio con similar información en la literatura. El modelo de elementos finitos permitió determinar la distribución y variación de los esfuerzos de Von Mises, los cuales aumentan cuando se presenta el síndrome de manguito rotador, con ruptura del tendón del supraespinoso. Dicho aumento se presentó en los puntos de inserción en
Aceptado: 12 de junio de 2015

los huesos y en la zona donde se presenta la ruptura, y se debe a la disminución del espesor de los tendones del manguito rotador.

Palabras clave: hombro, síndrome del manguito rotador, esfuerzos.

\section{ABSTRACT}

Syndrome rotator cuff shoulder pathology is most prevalent in Colombia, with the supraspinatus tendon most affected. The aim of this study is to develop a numerical model of the shoulder, to determine the variation of mechanical stress in a healthy shoulder, and in another with rotator cuff syndrome, because in Colombia there are no studies to determine such efforts. A model was developed in three dimensions of the shoulder, from a set of computed tomography images. This model includes the humerus, scapula, glenohumeral ligament, rotator cuff tendons and articular cartilage. Models were analyzed using the finite element method, and the Von Mises stress is obtained. Subsequently, the results of a healthy shoulder were compared, and a shoulder rotator cuff syndrome, for how varies the distribution of mechanical stress. Then the results obtained in this study with similar data in the literature were compared. The finite element model allowed to determine the distribution and variation of the Von Mises stress, which

\footnotetext{
- Docente investigador, Ing. Mecánica. Fundación Universidad de América. hector.castro@investigadores.uamerica.edu.co •- Estudiante coinvestigador, Ing. Mecánica.

-.. Estudiante coinvestigador, Ing. Mecánica.
} 
LÍNEA DE INVESTIGACIÓN: MODELADO COMPUTACIONAL

increases when the rotator cuff syndrome presents with rupture of the supraspinatus tendon. This increase was presented at the point of insertion in the bones and in the area where the break occurs, and is due to the thinning of the rotator cuff tendons.

Keywords: Shoulder, rotator cuff tear, stress.

\section{INTRODUCCIÓN}

El manguito rotador es un conjunto anatómico formado por los tendones de los músculos subescapular, infraespinoso, supraespinoso y teres menor (Cailliet). El síndrome del manguito rotador se manifiesta con dolor, y con la desestabilización funcional de la articulación glenohumeral (Terrier et al., 2007), y puede afectar a uno o todos los tendones de esta estructura anatómica, siendo el tendón del supraespinoso el más afectado. Desde un punto de vista biomecánico, las causas de esta enfermedad son las cargas, traumatismos previos, los movimientos repetitivos, o por encima del nivel del hombro (Vern Putz., 1997).

Ante la aparición de patologías como el síndrome del manguito rotador, se presentan aumentos en los esfuerzos mecánicos del hombro. En estudios realizados por autores de otros países, se han hallado los esfuerzos mecánicos en hombros sanos, con artroplastia (Hopkins et al., 2006; D'Amico et al., 2007) y con patologias como desgarros del manguito rotador (Steenbrink, 2006). Para determinar las cargas que se aplican al modelo geométrico del hombro, se han utilizado diferentes técnicas; por ejemplo, modelos artificiales para determinar las líneas de acción de los músculos y los momentos del brazo. En dichos modelos, los huesos se reemplazan por réplicas a escala hechas en resina epóxica (Favre et al., 2005) y los músculos y tendones se reemplazan por cuerdas. Otra manera de hallar las cargas, es considerar que la fuerza del músculo es proporcional al área de sección transversal fisiológica del mismo (PCSA por sus siglas en inglés), asumiendo que un músculo que tiene una sección transversal ma- yor, ejerce fuerzas de mayor magnitud (Bassett et al., 1990; De Luca and Forrest, 1973; Fick, 1850; Murray et al., 2000). Otros autores han usado técnicas como la electromiografía, la cual registra la actividad eléctrica de los músculos (Koike and Kawato, 1995; Langenderfer et al., 2005; Laursen et al., 1998). La fuerza del músculo es estimada asumiendo una relación lineal entre la actividad eléctrica y la fuerza bajo condiciones isométricas. Dichas cargas se aplican en modelos geométricos, los cuales se obtuvieron a través de tomografías o de mediciones in vivo (D'Amico et al., 2007). Algunos modelos de elementos finitos, consideran los huesos como sólidos rígidos, y la articulación glenohumeral como una unión de tipo ball-and-socket (Favre et al, 2005; Van Der Helm, 1994; Yanagawa et al 2008; Terrier et al, 2007).

El objetivo de este artículo es desarrollar un modelo en tres dimensiones de elementos finitos, del hombro sano y del hombro con síndrome del manguito rotador, para determinar la variación en la distribución de los esfuerzos mecánicos, durante los movimientos de abducción y flexión. El síndrome del manguito rotador es la patología de hombro más frecuente en Colombia (Terrier, 2007), y sin embargo no se han realizado estudios que permitan determinar la variación en la distribución de los esfuerzos mecánicos del hombro, cuando se presenta esta patología.

\section{MÉTODOS}

A través de una tomografía axial computarizada, tomada de la base de datos de El Carver College of Medicine de la Universidad de lowa, se reconstruyó la geometría del húmero y la escápula, obteniendo como resultado los huesos como sólidos. Después, estos huesos se ensamblaron en abducción a 45 y $75^{\circ}$, y en flexión a 90 y $120^{\circ}$ (Bergmann et al., 2007). Se tuvo en cuenta la rotación de la escápula y del húmero en dichas posiciones para estos ensambles. Luego se agregaron, por cada ensamble, el cartílago articular del húmero, los tendones 
del manguito rotador y el ligamento glenohumeral. Este ligamento se modeló porque, bio-mecánicamente, brinda estabilidad a la articulación glenohumeral (Norkin, 2006). El cartílago articular fue generado como un sólido de 1,2 mm de espesor (Fox et al., 2008) y un radio de $27,2 \mathrm{~mm}$ (Kelkar et al., 2001), con áreas de contacto crea-

Figura 1. Modelo del hombro en abducción a $45^{\circ}$.

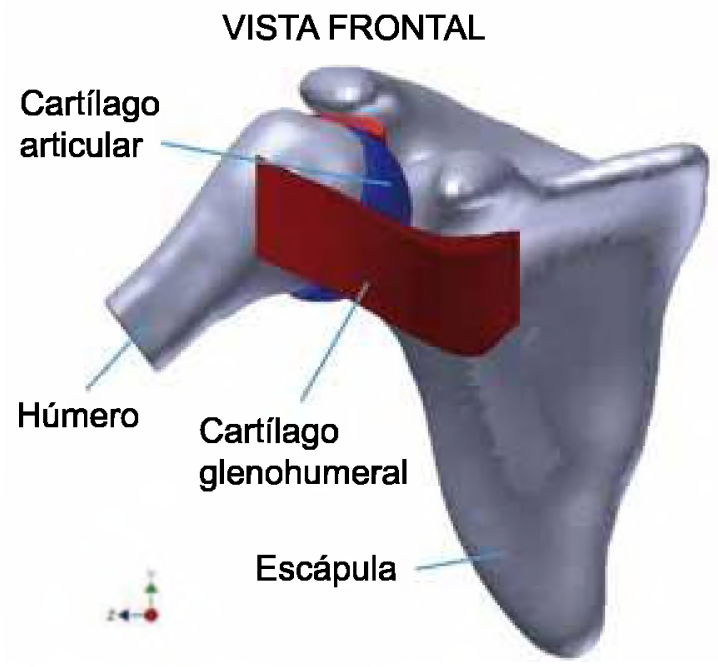

Fuente: los autores.

\section{Modelo geométrico del hombro}

Se tuvieron en cuenta los principales músculos que actúan en la abducción y la flexión, y los músculos cuyos tendones pertenecen al manguito rotador (Cailliet). Dichos músculos son: deltoides anterior, deltoides medio, deltoides posterior, teres menor, subescapular, infraespinoso y supraespinoso (Ver Figura 2). Las fuerzas de dichos músculos se obtuvieron de un modelo de hombro virtual desarrollado por OpenSim, una plataforma para modelar, simular y analizar sistemas neuromusculoesqueléticos (Opensim, en línea). Dicho modelo fue desarrollado de un cadáver de sexo masculino, y 1,70 $\mathrm{m}$ de altura (Holzbaur et al., 2005). El modelo de OpenSim proporciona las fuerzas de los músculos dependiendo de la posición, en función de los siguientes parámetros: la fuerza pico, el área de sección transversal fisiológica (PCSA), la longi- das en función de las superficies de los huesos. El ligamento glenohumeral y los tendones del manguito rotador, fueron modelados aproximando los puntos de inserción en los huesos (Terrier et al., 2006) con un espesor de $12 \mathrm{~mm}$ en los tendones y el ligamento (Papalia et al., 2011; De Gast, 1996). (Ver Figura 1).

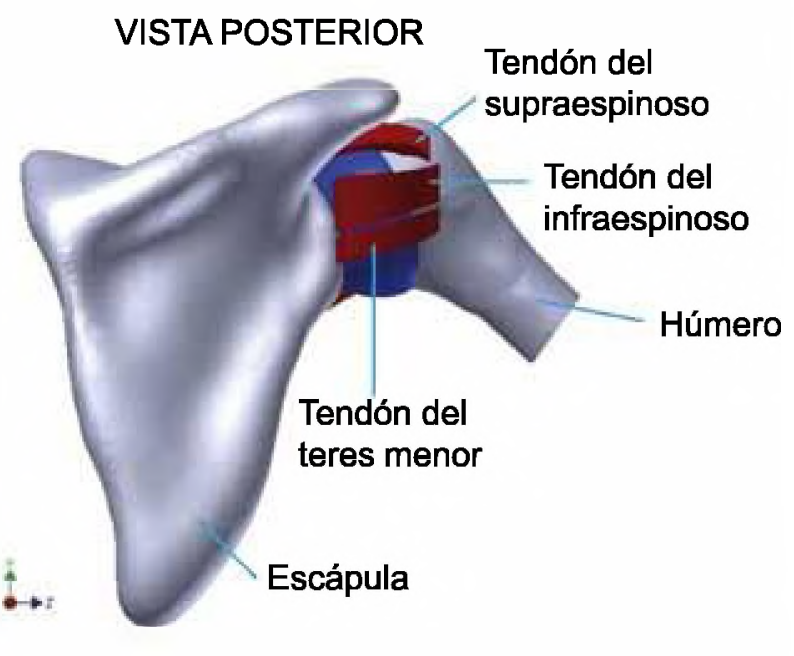

tud óptima de fibra y tendón, y el momento del brazo.

En la tabla 1 se muestra las fuerzas de cada músculo para las posiciones en abducción y flexión. Debido a que la carga se trasladó al punto de corte del húmero, se generó una fuerza y un momento equivalente. Dicha carga pasa por el centro de la cabeza del húmero y tiene en cuenta el peso del brazo, ya que está definido en el modelo virtual, y el momento se opone al movimiento (Bergmann et al., 2007). Las cargas resultantes están dentro del rango de fuerzas fisiológicas, que inciden en la articulación glenohumeral, comprendido de 930 a 1720 N (Virani et al., 2008).

Las propiedades mecánicas de los tejidos del hombro fueron modeladas como materiales lineales, elásticos e isotrópicos, como se muestra 
LÍNEA DE INVESTIGACIÓN: MODELADO COMPUTACIONAL

en la tabla 2. En las simulaciones, se usaron las propiedades de hueso cortical, el cual es denso, compacto, se encuentra en el cuerpo de los huesos largos y constituye aproximadamente el $80 \%$ del esqueleto. El módulo de Young de un hueso cortical puede llegar hasta los 18000 MPa (Buroni et al., 2004).

En la escápula se aplicó una restricción fija, la cual restringe la traslación y la rotación de este hueso, debido a que el ensamble conside- ra la rotación en el plano frontal, causada por los movimientos de abducción y flexión. Las estructuras como el tórax, los músculos y la piel restringen la rotación y traslación en los demás planos (Ver Figura 3).

En la figura 3, se muestra el punto de corte del húmero, en donde se aplicó la carga y el momento resultante. También se muestra la restricción fija aplicada en la escápula, la cual restringe la rotación y traslación.

Figura 2. Músculos de OpenSim.
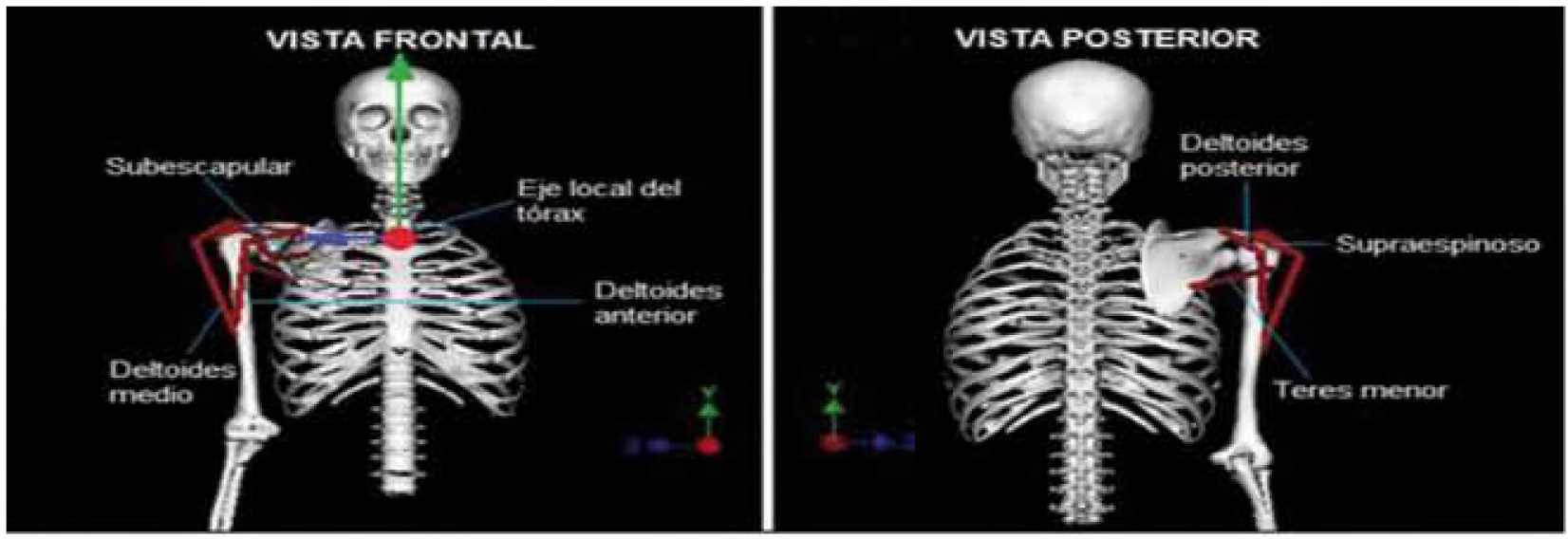

Fuente: Los autores. Modelo de OpenSim en el que se muestran los músculos seleccionados para determinar las cargas del modelo.

Tabla 1. Fuerzas de los músculos en abducción y flexión.

\begin{tabular}{|l|c|c|c|c|}
\hline \multicolumn{1}{|c|}{ Músculo } & $\begin{array}{c}\text { Fuerza en } \\
\text { abducción a 45 }\end{array}$ & $\begin{array}{c}\text { Fuerza en } \\
\text { abducción a } 75^{\circ}\end{array}$ & $\begin{array}{c}\text { Fuerza en flexión } \\
\text { a } 90^{\circ}\end{array}$ & $\begin{array}{c}\text { Fuerza en flexión } \\
\text { a } 120^{\circ}\end{array}$ \\
\hline Deltoides anterior & 0 & 1041,158 & $-504,249$ & 524,61 \\
\hline Deltoides medio & $-1060,686$ & $-865,564$ & $-802,656$ & $-807,066$ \\
\hline Deltoides posterior & $-242,138$ & 240,729 & $-193,603$ & 272,463 \\
\hline Supraespinoso & 459,444 & 0 & 0 & 484,293 \\
\hline Infraespinoso & $-1300,031$ & $-1082,1$ & $-1099,842$ & $-1378,246$ \\
\hline Teres menor & 343,099 & 353,125 & 333,29 & 0 \\
\hline
\end{tabular}

Fuente: Los autores. 
Tabla 2. Propiedades mecánicas de los tejidos.

\begin{tabular}{|l|c|c|l|}
\hline \multicolumn{1}{|c|}{ Estructura } & $\begin{array}{c}\text { Coeficiente de } \\
\text { Poisson }\end{array}$ & Módulo de Young (MPa) & \multicolumn{1}{|c|}{ Referencias } \\
\hline Hueso cortical & 0,3 & 18000 & Favre; Senteler et al; Sudipto et al \\
\hline Cartílago & 0,46 & 10,35 & Peña et al; Guo et al \\
\hline Ligamento & 0,4 & 62,63 & Ticker et al; Ellis; Debski et al \\
\hline
\end{tabular}

Fuente: Los autores.

Figura 3. Fuerza, momento y restricción del modelo.

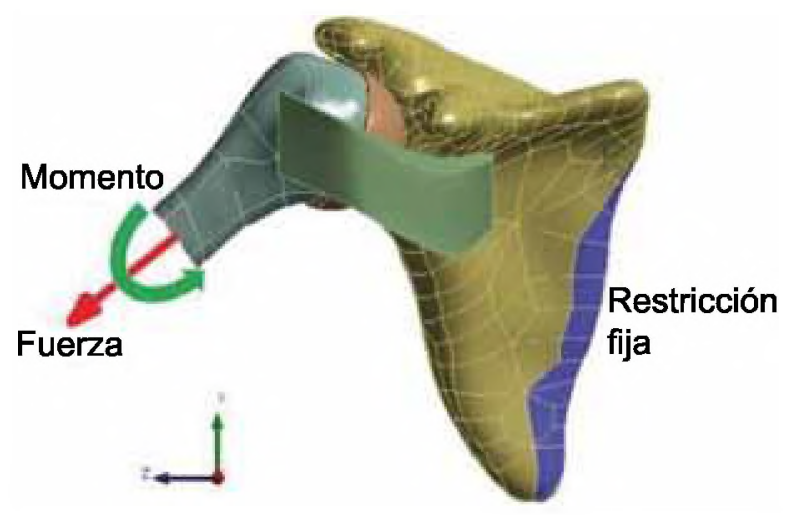

Fuente: Los autores.

\section{RESULTADOS}

Se hallaron los esfuerzos de Von Mises en el hombro en abducción a 45 y $75^{\circ}$ (Ver Tabla 3), y en flexión a 90 y $120^{\circ}$ (Ver Tabla 4). Al comparar los esfuerzos obtenidos en las estructuras de un hombro sano, con las del hombro con síndrome del manguito rotador, en abducción a $45^{\circ}$, se observó que el húmero no tiene variación en los esfuerzos, mientras que la escápula presentó un aumento del $0,73 \%$. El cartílago presentó un aumento del $8 \%$, el ligamento glenohumeral del $1,5 \%$, y los tendones del manguito rotador del $124 \%$. En abducción a $75^{\circ}$ los huesos no presentaron variación en los esfuerzos, mientras que el cartílago presentó un aumento del $21,3 \%$, el ligamento glenohumeral del $2 \%$ y los tendones del manguito rotador de $24 \%$. En la figura 4 se observa la distribución de los esfuerzos mecánicos en abducción a 45 y $75^{\circ}$.
En flexión a $90^{\circ}$, no se presentan variaciones en los esfuerzos del húmero, la escápula y el ligamento glenohumeral. El cartílago presenta un aumento de $40,4 \%$ y los tendones del manguito rotador de $21,4 \%$. En flexión a $120^{\circ}$ no hay variaciones en los esfuerzos del húmero, la escápula y el cartílago. El ligamento glenohumeral presenta un aumento del $2,3 \%$ y los tendones del manguito rotador de $45 \%$. En la figura 5 se muestra la distribución de los esfuerzos mecánicos en flexión a 90 y $120^{\circ}$.

\section{CONCLUSIONES}

\section{Discusión}

Se realizó un modelo del hombro sano y con síndrome del manguito rotador con ruptura total del tendón del supraespinoso. Luego, se determinaron las cargas y el momento resultantes al cortar el húmero y trasladar las cargas de cada músculo a este punto; y se determinaron las propiedades mecánicas de los tejidos. Posteriormente, se realizó un análisis de elementos finitos para hallar la distribución de los esfuerzos de Von Mises en la articulación del hombro.

Este modelo de hombro provee aproximaciones útiles del comportamiento de los esfuerzos de las estructuras analizadas. Los esfuerzos obtenidos en este estudio, para la escápula, están en un rango de 0 a $57 \mathrm{MPa}$., los cuales están dentro del rango de esfuerzos reportados por otros autores, que va de 0 a $65 \mathrm{MPa}$. (Gupta et al., 2004). Los esfuerzos obtenidos en el ligamento glenohumeral están entre 0 y $14 \mathrm{MPa}$., 
LÍNEA DE INVESTIGACIÓN: MODELADO COMPUTACIONAL

y los reportados en la literatura están entre 0 y $7 \mathrm{MPa}$. (Debski et al., 2005). Los esfuerzos obtenidos en el cartílago están entre 0 y 4 MPa., y los esfuerzos reportados en otros estudios están entre 0 y $2 \mathrm{MPa}$. (Favre., 2011). Se evidencia que cuando se padece el síndrome del manguito rotador, se presentan aumentos considerables en los esfuerzos mecánicos en todas las estructuras del hombro, excepto en los huesos. Las concentraciones más altas de esfuerzos se presentan en los puntos de inserción de los tejidos en los huesos, y en el sitio de la ruptura del tendón del supraespinoso. Este aumento se debe a la ruptura de este tendón, lo que conlleva a una disminución del área de soporte.

El modelo tiene algunas limitaciones: el ligamento glenohumeral, los tendones del manguito rotador y el cartílago, son materiales no lineales. Sin embargo, la aproximación de material lineal, elástico e isotrópico, es una buena aproximación. No se tuvieron en cuenta todas las estructuras de la articulación del hombro, las cuales podrían ser analizadas.

Se creó un modelo que permitió determinar la distribución de los esfuerzos mecánicos en un hombro, cuando presenta el síndrome del manguito rotador, para posteriormente cuantificar en qué porcentaje varían los esfuerzos. Éstos aumentan, en comparación a un hombro sano, debido a la pérdida de material del tendón, lo que reduce el área de sección transversal que soporta el esfuerzo general. En los resultados obtenidos en el hombro con síndrome del manguito rotador, se observa que los esfuerzos aumentan, en comparación con el hombro sano.

Figura 4. Distribución de los esfuerzos mecánicos en abducción a 45 y $75^{\circ}$.

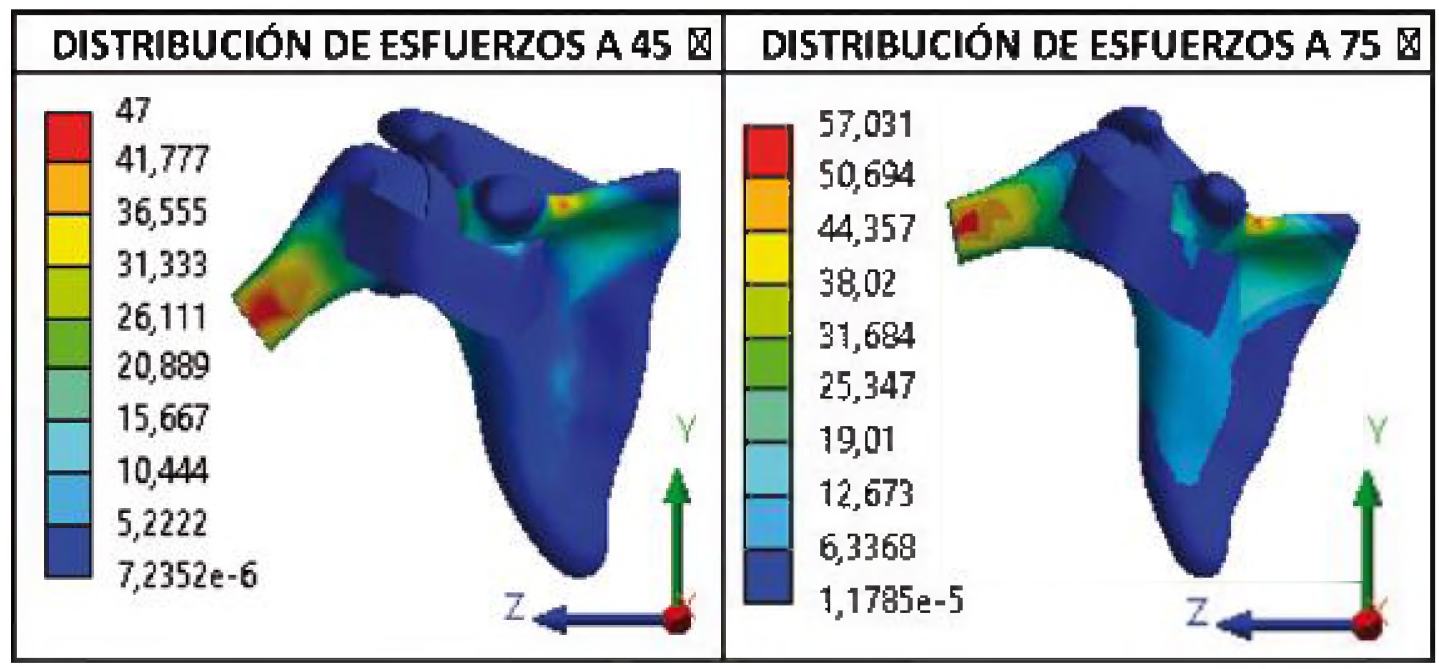

Fuente: Los autores. 
Tabla 3. Esfuerzos de Von Mises en abducción.

\begin{tabular}{|c|c|c|c|c|}
\hline \multicolumn{5}{|c|}{ Resultados en abducción } \\
\hline Angulo & & $45^{\circ}$ & & $5^{\circ}$ \\
\hline Componente & $\begin{array}{l}\text { - Von Mises } \\
\text { Sano (Mpa) }\end{array}$ & $\begin{array}{c}\sigma \text { V Von Mises } \\
\text { Sindrome del } \\
\text { manguito rotador } \\
\text { (Mpa) }\end{array}$ & $\begin{array}{l}\sigma \text { Von Mises } \\
\text { Sano (Mpa) }\end{array}$ & $\begin{array}{c}\text { o Von Mises } \\
\text { Síndrome del } \\
\text { manguito rotador } \\
\text { (Mpa) }\end{array}$ \\
\hline Húmero & 47 & 46,999 & 52,785 & 52,786 \\
\hline Escápula & 43,345 & 43,661 & 57,031 & 57,265 \\
\hline Cartílago & 2,6578 & 2,8739 & 3,2241 & 3,91 \\
\hline Ligamento glonohumeral & 14,218 & 14,439 & 11,991 & 12,246 \\
\hline Manguito rotador & 10,746 & 24,116 & 20,508 & 25,447 \\
\hline
\end{tabular}

Fuente: Los autores.

Figura 5. Distribución de los esfuerzos mecánicos en flexión a 90 y $120^{\circ}$.

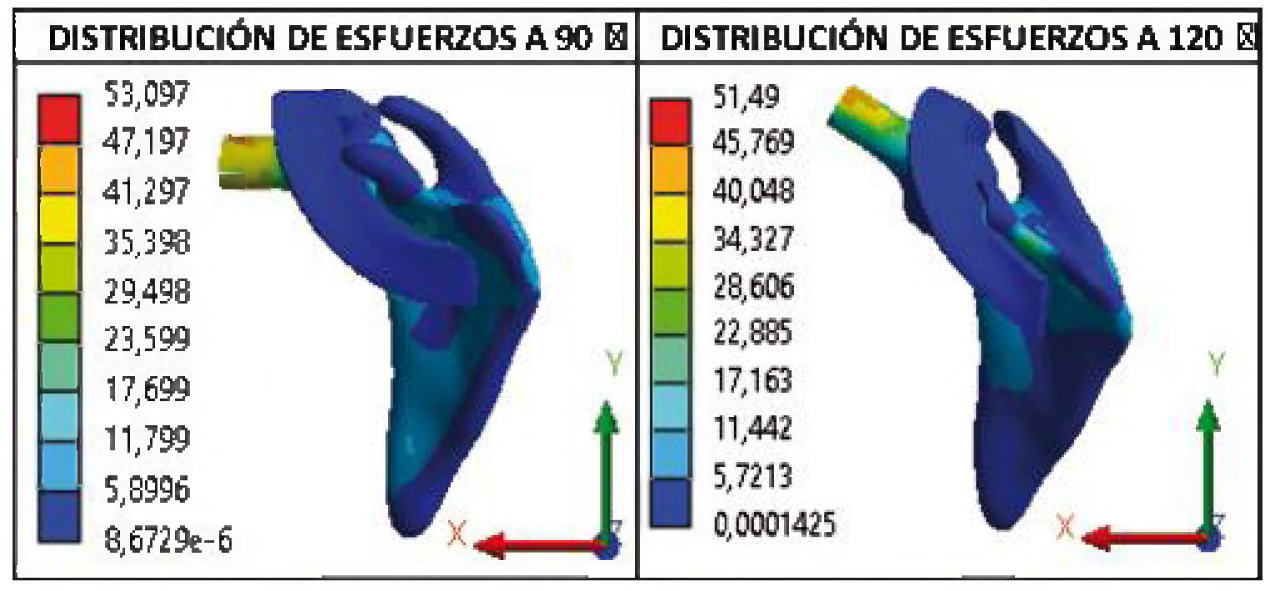

Fuente: Los autores.

Tabla 4. Esfuerzos de Von Mises en flexión.

\begin{tabular}{|c|c|c|c|c|}
\hline \multicolumn{5}{|c|}{ Resultados en abducción } \\
\hline Angulo & \multicolumn{2}{|c|}{$90^{\circ}$} & \multicolumn{2}{|c|}{$120^{\circ}$} \\
\hline Componente & $\begin{array}{l}\text { o Von Mises } \\
\text { Sano (Mpa) }\end{array}$ & $\begin{array}{c}\sigma \text { Von Mises } \\
\text { Sindrome del } \\
\text { manguito rotador } \\
\text { (Mpa) }\end{array}$ & $\begin{array}{l}\sigma \text { Von Mises } \\
\text { Sano (Mpa) }\end{array}$ & $\begin{array}{c}\sigma \text { Von Mises } \\
\text { Sindrome del } \\
\text { manguito rotador } \\
\text { (Mpa) }\end{array}$ \\
\hline Húmero & 53,097 & 53,097 & 51,49 & 51,49 \\
\hline Escápula & 36,08 & 36,067 & 34,963 & 35,029 \\
\hline Cartílago & 4,3293 & 6,0782 & 4,4894 & 4,538 \\
\hline Ligamento glonohumeral & 8,64 & 8,652 & 14,159 & 14,496 \\
\hline Manguito rotador & 14,467 & 17,57 & 13,621 & 19,783 \\
\hline
\end{tabular}

Fuente: Los autores. 


\section{REFERENCIAS}

Bassett, R.; Browne, W.; Morrey, W.; An, F.; (1990) “Glenohumeral muscle force and moment mechanics in a position of shoulder instability". J. biomech 23, 1990. P. 405-15

Bergmann, G., Graichen, F.; Bender, A.; Kääb, M.; Rohlmann, A.; Westerhoft, P. (2007) "In vivo: glenohumeral contact forces-measurements in the first patient 7 months postoperatively". Journal of biomechanics. Vol. 40 , no $10, \mathrm{p}$. 2139-2149.

Buroni, C.; Commisso, E.; Cisilino, P. \& Sammartino, M. (2004) "Determinación de las constantes elásticas anisótropas del tejido óseo utilizando tomografías computadas. Aplicación a la construcción de modelos de elementos finitos". Mecánica computacional, 23. P. 3009-3032

Cailliet, R. (2006)." Anatomía funcional, biomecánica". Marbán. P. 113.

Cisilino, A., D’Amico, D., Buroni, F., Commisso, P., Sammartino, M., \& Capiel, C. (2008). "Construcción de modelos computacionales para el análisis de esfuerzos mecánicos de piezas óseas utilizando imágenes de TC: aplicación a la articulación gleno-humeral". Revista argentina de radiología, 72(4), 443-448.

de Gast, A. (1996). “Normal and impaired mobility of the glenohumeral joint" (Doctoral dissertation, PhD Thesis, Copynomie, Rotterdam). P. 25.

de Duca, C. J., \& Forrest, W. J. (1973). "Force analysis of individual muscles acting simultaneously on the shoulder joint during isometric abduction". Journal of biomechanics, 6(4), 385-393.

Debski, R. E., Weiss, J. A., Newman, W. J., Moore, S. M., \& McMahon, P. J. (2005). "Stress and strain in the anterior band of the inferior glenohumeral ligament during a simulated clinical examination". Journal of shoulder and elbow surgery, 14(1), S24-S31.

Favre, P. (2011). “A model to study active shoulder motion and stability" (Doctoral dissertation, Eth Zurich). P. 59.

Favre, P., Sheikh, R., Fucentese, S. F., \& Jacob, H. A. (2005). "An algorithm for estimation of shoulder muscle forces for clinical use". Clinical Biomechanics, 20(8), 822833.

Fick, A. (1850). "Statische Betrachtung der muskulatur des Oberschenkels".Zeitschr. $f$. rat. Med, 9, 1849.

Fox, J. A., Cole, B. J., Romeo, A. A., Meininger, A. K., Glenn Jr, R. E., Bicos, J., ... \& Dorow, C. B. (2008). "Articular cartilage thickness of the humeral head: an anatomic study". Orthopedics, 31(3), 216.
Gupta, S., \& van der Helm, F. C. (2004). "Load transfer across the scapula during humeral abduction". Journal of biomechanics, 37(7), 1001-1009.

Holzbaur, K. R., Murray, W. M., \& Delp, S. L. (2005). "A model of the upper extremity for simulating musculoskeletal surgery and analyzing neuromuscular control". Annals of biomedical engineering, 33(6), 829-840.

Hopkins, A. R., Hansen, U. N., Amis, A. A., Taylor, M., Gronau, N., \& Anglin, C. (2006). "Finite element modelling of glenohumeral kinematics following total shoulder arthroplasty". Journal of biomechanics, 39(13), 2476-2483.

Kelkar, R., Wang, V. M., Flatow, E. L., Newton, P. M., Ateshian, G. A., Bigliani, L. U., ... \& Mow, V. C. (2001). "Glenohumeral mechanics: a study of articular geometry, contact, and kinematics". Journal of Shoulder and Elbow Surgery, 10(1), 73-84.

Koike, Y., \& Kawato, M. (1995). "Estimation of dynamic joint torques and trajectory formation from surface electromyography signals using a neural network model". Biological cybernetics, 73(4), 291-300.

Langenderfer, J. E., Patthanacharoenphon, C., Carpenter, J. E., \& Hughes, R. E. (2006). "Variation in external rotation moment arms among subregions of supraspinatus, 
infraspinatus, and teres minor muscles". Journal of orthopaedic research, 24(8), 1737-1744.

Laursen, B., Jensen, B. R., Németh, G., \& Sjøgaard, G. (1998). "A model predicting individual shoulder muscle forces based on relationship between electromyographic and 3D external forces in static position". Journal of biomechanics, 31(8), 731-739.

Murray, W. M., Buchanan, T. S., \& Delp, S. L. (2000). "The isometric functional capacity of muscles that cross the elbow". Journal of biomechanics, 33(8), 943-952.

Norkin, C. C., \& White, D. J. (2006). "Goniometría: evaluación de la movilidad articular". Marbán. p. 57.

Opensim, en linea: https:/l simtk.org/home/opensim. Consultado el 25 de agosto de 2014

Papalia, R., Franceschi, F., Del Buono, A., Maffulli, N., \& Denaro, V. (2011). "Results of surgical management of symptomatic shoulders with partial thickness tears of the rotator cuff". British medical bulletin, 99(1), 141-154.

Bernard, B. P. (Ed.). (1997). "Musculoskeletal disorders and workplace factors: a critical review of epidemiologic evidence for work-related musculoskeletal disorders of the neck, upper extremity, and low back" (No. 97-141). US Department of Health and Human Services, Public Health Service, Centers for Disease Control and Prevention, National Institute for Occupational Safety and Health.

Steenbrink, F., de Groot, J. H., Veeger, H. E. J., Meskers, C. G. M., van de Sande, M. A. J., \& Rozing, P. M. (2006). "Pathological muscle activation patterns in patients with massive rotator cuff tears, with and without subacromial anaesthetics". Manual therapy, 11(3), 231-237.

Terrier, A., Büchler, P., \& Farron, A. (2006). "Influence of glenohumeral conformity on glenoid stresses after total shoulder arthroplasty". Journal of shoulder and elbow surgery, 15(4), 515-520.
Terrier, A., Reist, A., Vogel, A., \& Farron, A. (2007). "Effect of supraspinatus deficiency on humerus translation and glenohumeral contact force during abduction". Clinical Biomechanics, 22(6), 645-651.

Van der Helm, F. C. (1994). "A finite element musculoskeletal model of the shoulder mechanism". Journal of biomechanics, 27(5), 551-569.

Virani, N. A., Harman, M., Li, K., Levy, J., Pupello, D. R., \& Frankle, M. A. (2008). "In vitro and finite element analysis of glenoid bone/baseplate interaction in the reverse shoulder design". Journal of Shoulder and Elbow Surgery, 17(3), 509521.

Yanagawa, T., Goodwin, C. J., Shelburne, K. B., Giphart, J. E., Torry, M. R., \& Pandy, M. G. (2008). "Contributions of the individual muscles of the shoulder to glenohumeral joint stability during abduction". Journal of biomechanical engineering, 130(2), 021024. 